\title{
Design and Optimization of an Autonomous, Ambulatory Cardiac Event Monitor
}

\author{
Bertrand Massot \\ INL, CNRS UMR5270 \\ Univ. Lyon, INSA Lyon \\ Villeurbanne, France \\ bertrand.massot@insa-lyon.fr
}

\author{
Florin Hutu \\ INRIA, CITI \\ Univ. Lyon, INSA Lyon \\ Villeurbanne, France \\ florin-doru.hutu@insa-lyon.fr
}

\author{
Claudine Gehin \\ INL, CNRS UMR5270 \\ Univ. Lyon, INSA Lyon \\ Villeurbanne, France \\ claudine.gehin@insa-lyon.fr
}

\author{
Norbert Noury \\ INL, CNRS UMR5270 \\ Univ. Lyon, UCBL \\ Villeurbanne, France \\ norbert.noury@insa-lyon.fr
}

\begin{abstract}
Wearable sensors for health monitoring can enable the early detection of various symptoms, and hence rapid remedial actions may be undertaken. In particular, the monitoring of cardiac events by using such wearable sensors can provide realtime and more relevant diagnosis of cardiac arrhythmias than classical solutions. However, such devices usually use batteries, which require regular recharging to ensure long-term measurements. We therefore designed and evaluated a connected sensor for the ambulatory monitoring of cardiac events, which can be used as an autonomous device without the need of a battery. Even when using off-the-shelf, low-cost integrated circuits, by optimizing both the hardware and software embedded in the device, we were able to reduce the energy consumption of the entire system to below $0.4 \mathrm{~mW}$ while measuring and storing the ECG on a non-volatile memory. Moreover, in this paper, a power-management circuit able to store energy collected from the radio communication interface is proposed, able to make the connected sensor fully autonomous. Initial results show that this sensor could be suitable for a truly continuous and long-term monitoring of cardiac events.
\end{abstract}

Index Terms-wearable sensors, cardiac event monitor, electrocardiography, autonomous systems.

\section{INTRODUCTION}

Cardiovascular diseases are the leading cause of death in the world, and the global rate has been constantly increasing over the past decades (from $25.8 \%$ in 1990 to $32.1 \%$ in 2015) [1]. Electrocardiograms (ECG) are non-invasive recordings of the electrical activity of the heart which enable the diagnosis of cardiovascular diseases through the analysis of the waveform [2]-[6]. Moreover, early symptoms of cardiac diseases, such as arrhythmias or myocardial ischemia, can be detected directly by monitoring the electrocardiogram [7], [8]. However, it is obviously not possible for an at-risk patient to take up residence in their doctor's clinic while waiting to catch the early symptoms of a cardiac event. A more convenient way of early detection would be to wear a small, portable device, which monitors and automatically detects abnormalities in the ECG [9]. Unfortunately, these devices require either large batteries or frequent recharging as the device needs to continuously monitor the vital signal over a long period.

Nowadays, the most common way of recording - and subsequently analyzing - the electrocardiogram out of the

This work was supported by the Institut National des Sciences Appliquées de Lyon under BQR 2015-2017 program. hospital is by means of ECG recorders called "Holters". Holters are mobile, body-worn electrocardiograph devices, which are usually worn for more than 24 hours. This continuous period of recording can indeed be useful in observing occasional/intermittent heart rhythm disorders. Due to the unpredictable nature of abnormalities in heart rate and cardiac arrhythmias, these tend not to be readily detected during short medical examinations. For longer periods of medical supervision (i.e. months), standard continuous monitors fail to record the entire ECG due to a lack of sufficient memory or autonomy (or because the monitored period is too long to be subsequently analyzed manually). Instead, specific Holters called "Cardiac Event Monitors" or "R-test" can be worn to intermittently record, when appropriate, key short episodes of the ECG over the extended monitoring period. Patients suffering from heart rhythm disorders often sense cardiac arrhythmias through associated pains or dizziness. They can then press a button on the worn device to trigger the recording of several seconds of ECG at that time, which will be later conveniently analyzed by a specialist.

In this paper, we propose a cardiac event monitor system, which has been thoroughly optimized in terms of circuit design, as well as software development, in order to achieve ultra-low power consumption and thus make it suitable for long-term, autonomous operation using the harvested energy. The sensor system is attached on the chest of the patient using disposable, adhesive electrodes or, more conveniently, using a chest belt with three dry electrodes, to record a single lead electrocardiogram. By pressing a button on the device, a short period of the ECG is recorded on an embedded, non-volatile memory. The signal can then be retrieved via a smartphone through near-field wireless communication (NFC). In order to decrease the device's overall energy consumption at the lowest possible level, the microcontroller of this system was selected and programmed to obtain the best compromise between power consumption and other inherent constraints linked to the targeted medical application.

The rest of the paper is structured as follows: in section II, we describe the overall architecture, as well as the various functions to be fulfilled by the device and their impact on the design. In particular, a comparative analysis of various microcontrollers is presented, under measurement conditions, 
which correspond to the envisaged application. The specific peripherals' power management, the analog front-end and the memory interface are also presented. For a better form factor and housing of the sensor, the routing of the circuit was made within the NFC antenna area. Optimizations made on the antenna to achieve good impedance matching and thus, a maximum RF power transfer are presented in section III. Experiments conducted to characterize device performances, as well as the obtained results are described in section IV. Finally, the preliminary design of a module connected to the sensor system that is able to store the energy collected from the NFC interface and then power back the sensor board, thus turning the system into a fully autonomous sensing device is presented.

\section{CONNECTED SENSOR'S ARCHITECTURE AND OPERATION}

\section{A. Analysis of microcontrollers' power consumption}

The appropriate microcontroller selection resulted from a comparative analysis of power consumption, that was performed on three components that could possibly match the constraints of the application (cf. list below). Regarding the energy consumption optimization of the overall device, this step is of paramount importance as it cannot be reliably assessed solely on the electrical specifications as described in the technical documents provided by the manufacturer. For example, the lowest power consumption quoted in the documentation can be generally only be achieved under specific conditions, which are not necessarily those of the proposed application in this paper. Consequently, the selected microcontrollers were tested against the specific static and dynamic conditions of the present application. For this analysis, three microcontrollers were selected for assessment, based on their quoted specifications:

- CY8C4045 (PSoC400S), Cypress Semiconductors [10];

- STM8L152C6, STMicroelectronics [11];

- MSP430FR5969, Texas Instrument [12].

While essentially different in terms of device family, manufacturer, and type (8, 16 and 32-bit MCUs), these three MCU were chosen according to common specifications:

- A Real-Time Calendar (RTC) function, necessary to keep track of the time at which the periods of ECG are recorded;

- An Inter-Integrated Circuit (I2C) serial bus, to enable communication of the MCU with the dual interface EEPROM;

- A 10-bit (minimum) ADC capable of sampling the ECG at a minimum of 100 samples per second;

- An active current consumption less than or equal to 200 $\mu \mathrm{A}$ per $\mathrm{MHz}$ (CPU operating frequency), a threshold which is representative of existing low-power MCUs;

- An idle current consumption at a microampere level.

According to their respective technical specifications, the expected current consumptions are reported in Table I.
TABLE I

COMPARISON OF CURRENT CONSUMPTION FOR MICROCONTROLLERS BASED ON TECHNICAL SPECIFICATIONS

\begin{tabular}{c|c|c|c} 
& CY8C4045AZI-S413 & STM8L152C6 & MSP430FR5969 \\
\hline Core & ARM Cortex M3 & STM8 & MSP430 \\
\hline Width & 32 bits & 8 bits & 16 bits \\
\hline Active & $96-200 \mu \mathrm{A} / \mathrm{MHz}$ & $180 \mu \mathrm{A} / \mathrm{MHz}$ & $100 \mu \mathrm{A} / \mathrm{MHz}$ \\
\hline Idle & $2.5 \mu \mathrm{A}$ & $0.25 \mu \mathrm{A}$ & $0.4 \mu \mathrm{A}$
\end{tabular}

Four different situations were chosen in order to assess the actual current consumption of the microcontrollers, classified as two steady and two transient situations. The first steady state is defined as the lowest power mode accessible by the microcontroller while keeping an active clock to maintain the RTC function working and the second one is defined as the lowest active mode with an operating frequency of $1 \mathrm{MHz}$. During the two transient scenarios, the variations in current and voltage were measured to assess both the energy used by the microcontroller to boot, and the energy needed for a single analog-to-digital conversion using the internal ADC. All the above situations were tested using two different supply voltages: $2.2 \mathrm{~V}$ and $3.3 \mathrm{~V}$. The former is the minimum voltage under which all the microcontrollers were able to boot and properly operate at $1 \mathrm{MHz}$, while the latter is a standard supply voltage for embedded devices.

During the steady situations, measurements were carried out using a source-meter (Keithley's Model 2400), configured as a voltage source while measuring an accurate value of the supplied current. For the transient situations, a shunt resistor was used to monitor the variations of the supply current. Moreover, the sensor's supply voltage was also recorded to compute the power as well as the energy over the measurement period. For this setup, a $10 \Omega$ shunt resistor was used to monitor the current. As the current itself was always less than $1 \mathrm{~mA}$, the voltage drop remained below $10 \mathrm{mV}$ which is negligible with respect to the original voltage supply of 2.2 and $3.3 \mathrm{~V}$. The voltages across the resistance and the sensor were acquired using differential analog channels of a digital scope (Digilent's Analog Discover 2) at a sampling rate of 800 $\mathrm{kHz}$. Care was given to remove all bypass capacitors when measuring the energy consumed during the boot sequence to avoid current peaks.

Results obtained under each of the situations are illustrated on Figure 1. As predicted from the technical specifications, 32-bit ARM Cortex M0+ core, while being the lowest power consuming MCUs from the ARM architectures, have the highest current consumption compared to the STM8L and MSP430 proprietary cores. Regarding the current consumption in active mode with an operating frequency of $1 \mathrm{MHz}$, the results are much higher than expected from specifications (of the order of $100 \mu \mathrm{A}$ at $1 \mathrm{MHz}$ ). This is explained by the way these MCUs are clocked internally: for the experiment, the internal oscillator of each MCU was used to clock the system, as they are usually low power and this saves place on the circuit 

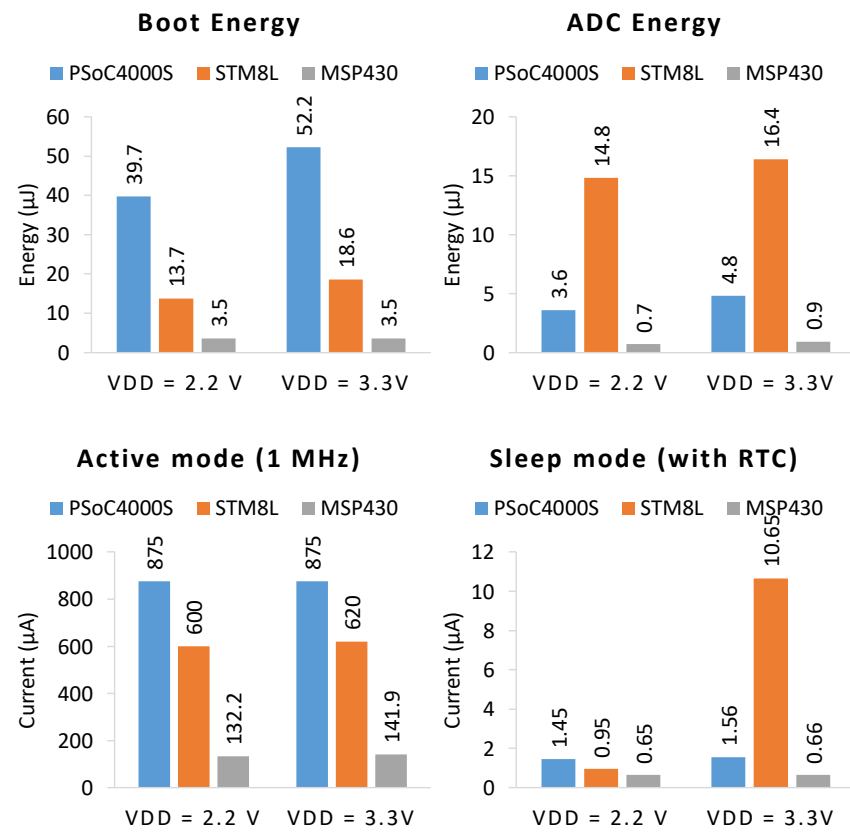

Fig. 1. Comparison of the energy and current consumptions for microcontrollers based on measurements under steady (idle and active modes) and transient states (at boot and during analog-to-digital conversion) with supply voltages of $2.2 \mathrm{~V}$ and $3.3 \mathrm{~V}$.

board. However, for the CY8C4045 and the STM8L152C6, the internal oscillators have a minimal frequency, which is higher than $1 \mathrm{MHz}$ (respectively 24 and $16 \mathrm{MHz}$ ). Consequently, the operating frequency of $1 \mathrm{MHz}$ is obtained using internal prescaler that divide the original clock down. As such, even if the final operating frequency is $1 \mathrm{MHz}$, the current consumption (mainly due to the frequency of the original clock) remains much higher than expected. The MSP430FR5969 was the only MCU which, in our experiment, had a current consumption below $100 \mu \mathrm{A}$ at an operating frequency of $1 \mathrm{MHz}$. We also found that the STM8L152C6 has an idle consumption which increases non-linearly with the voltage supply, up to $10 \mu \mathrm{A}$ at $3.3 \mathrm{~V}$. For the present application, the MSP430FR5969 was finally selected because of the lower energy required for a single analog-to-digital conversion as well as the almost constant current consumption over a wide voltage supply range $(2.2 \mathrm{~V}$ to $3.3 \mathrm{~V})$.

\section{B. Connected sensor's operation}

An overview of the sensor architecture is given on Figure 2. Power supply of the peripherals is directly controlled using GPIOs of the MCU, as described above. The analog frontend is connected to 3 electrodes on the individual for a single lead ECG measurement using a Driven Right-Leg circuit (DRL) which is an active feedback and greatly improves commonmode noise rejection in ambulatory ECG measurements [13]. Energy harvesting output and the main voltage supply of the circuit are connected to an external power management and energy storage circuit described in section V.

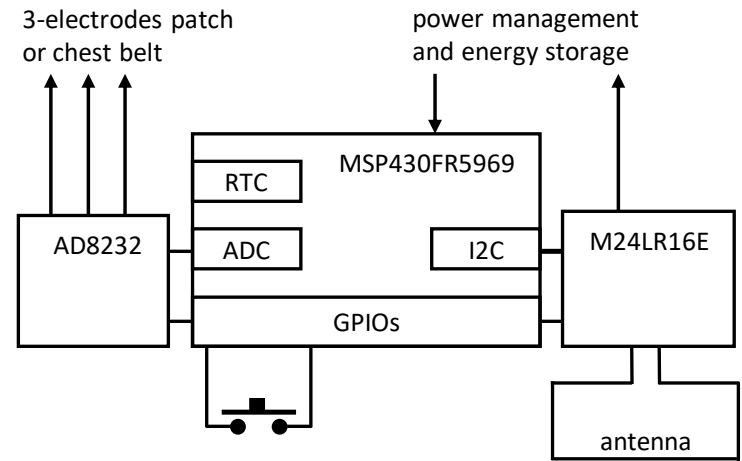

Fig. 2. Simplified schematic of the sensor circuit with connections to an external power management unit.

Apart during the measurement periods, the sensor remains in the lowest power state possible while keeping a low frequency clock active. This clock is based on a precise $32.768 \mathrm{kHz}$ crystal as it serves to keep track of time, by feeding the RTC function of the MSP430FR5969 MCU. In this state, the AD8232 as well as the M24LR16E are completely powered down, and the MCU is set to a low power, idle mode, with only low frequency clock active while all internal peripherals as well as the CPU remain disabled.

The system can be woken up only by the connected sensor's push button. Pressing this button switches the mode of the MCU from idle to active and triggers an interrupt to start the measurement of a single, 5-second segment of ECG. Beforehand, the analog front-end is powered up during 2 seconds in order to provide settling time to the analog filters, while the MCU is put back in low power mode. Then, a periodic timer with a period of $10 \mathrm{~ms}$ is set in the MCU so it is woken up with a rate of $100 \mathrm{~Hz}$ in order to acquire one sample from the output of the AD8232 using its internal, 12-bit ADC.

The samples are buffered in the MCU RAM until 500 samples have been acquired. The analog front-end is then powered down, and the whole buffer is written on the M24LR16E's EEPROM as along with the measurement timestamp, read from the RTC function. Then, the M24LR16E device is powered up and then the data is sent through the $\mathrm{I} 2 \mathrm{C}$ interface, whereupon it is powered down again and the whole system is set back in the initial state, waiting for a new measurement to be triggered via the push button.

\section{RF DESIGN CONSIDERATIONS}

The RF communications are reputed as one of the energy greedy parts of an embedded system and ultra-low energy communication protocols such as (Bluetooth Low Energy, Zigbee, Zwave, EnOcean, RFID/NFC, etc.) are on available. Moreover, strategies to further reduce the impact of the radio front-end on overall energy consumption are proposed in the scientific literature. These strategies are efficient, especially for low duty cycle scenarios, and consist in deactivating the radio interface during the standby periods. Synchronous wake-ups 
(driven by the MCU) or asynchronous ones (driven by quasipassive radio receivers or "wake-up radios") can be considered and energy consumption reductions in standby mode can be achieved.

For the present application, the NFC protocol was chosen not only because of its implementation simplicity, but also because on the sensor's side, the energy fingerprint of this kind of radio is positive, i.e. it is able to deliver energy to power the other peripherals of the sensor.

NFC is a wireless communication protocol for low range (several centimeters), high frequency (13.56 MHz) and low data rate communications. As discussed previously, between the solutions proposed on the market, the ST Microelectronics' M24LR16E [14] was chosen for implementation considerations. At the radio side, this is a dynamic NFC/RFID reader using ASK (amplitude shift keying) modulation and a pulse position coding of ratio $1 / 4$ or $1 / 256$ to achieve datarates from $1.6 \mathrm{kBit} / \mathrm{s}$ to $26 \mathrm{kBit} / \mathrm{s}$, fair enough for our application.

The communication range and the amount of harvested energy are strictly related to the impedance of the coil (or antenna) connected to the M24LR16E. From the manufacturer's datasheet, for an internal input capacitance of $27.5 \mathrm{pF}$, the ideal value of this impedance is $Z_{\text {ant }}=0+\jmath \cdot 426 \Omega$ which corresponds to a $5 \mu H$ inductance.

The antenna's design was performed by taking into account the dimensions of the initial circuit and the electromagnetic coupling between the circuit and the coil. The substrate used for the design and manufacturing of the antenna was the classical $1.6 \mathrm{~mm}$ FR4 having $\varepsilon_{r}=4.7$ and a dissipation factor of 0.014. Using the Keysight's ADS software, an optimization routine was implemented and so, the appropriate dimensions of a spiral coil with 9 turns were found. As can be seen on Figure 3 , the antenna is compact, surrounding the PCB containing the microcontroller, the NFC radio and the EEG front-end. The coil's input impedance obtained after optimization at 13.56 $\mathrm{MHz}$ was $Z_{\text {coil }}=2.15+\jmath \cdot 426 \Omega$, which is closed to the desired value.

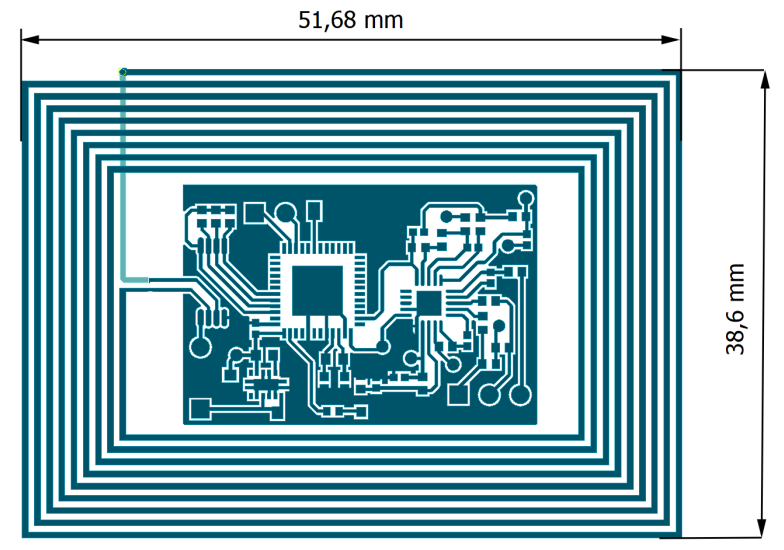

Fig. 3. Layout dimensions after optimization using Keysight's ADS Momentum

\section{EXPERIMENTAL RESULTS}

\section{A. Sensor's power consumption}

The connected sensor has been tested on a test-bench using the source-meter in order to assess consumption during its four different states: idle mode, settling, measuring, and memory write. The measurement results are presented in Table II. During idle mode, a minimum current of $730 \mathrm{nA}$ is required when powering the sensor with a $2.2 \mathrm{~V}$ voltage supply to keep the RTC function working. This current is slightly higher if the board is supplied at $3.3 \mathrm{~V}$. This means that, depending on the voltage supply, a minimal amount of power is required to keep the sensor in idle mode. At $2.2 \mathrm{~V}$, the power needed is $1.6 \mu \mathrm{W}$ while it increases up to $2.6 \mu \mathrm{W}$ when being operated at $3.3 \mathrm{~V}$, as the current drawn remains almost identical. If the energy is provided directly from a pre-charged capacitor, the voltage will linearly decrease down to $2.2 \mathrm{~V}$, at a rate depending on the capacitance $C$. Consequently, a minimum amount of energy, equal to $2.42 \times C$, must be stored permanently in the capacitor and will remain unused. This case is further discussed in section $\mathrm{V}$ as a solution is presented using a super-capacitor. On the other hand, if the sensor is supplied by using energy harvesting strategies, the generator must provide at least 1.6 $\mu \mathrm{W}$ at all times.

TABLE II

POWER CONSUMPTION AND ENERGY REQUIREMENTS

\begin{tabular}{lccccc}
\hline & \multicolumn{2}{c}{ Current $(\mu \mathrm{A})$} & & \multicolumn{2}{c}{ Energy $(\mathrm{mJ})$} \\
\cline { 2 - 3 } Mode & $2.2 \mathrm{~V}$ & $3.3 \mathrm{~V}$ & Duration (s) & $2.2 \mathrm{~V}$ & $3.3 \mathrm{~V}$ \\
\hline Idle & 0.73 & 0.79 & - & - & - \\
Settling & 150 & 155 & 2 & 0.66 & 1.02 \\
Measure & 157 & 162 & 5 & 1.73 & 2.67 \\
Write & 160 & 200 & 1.2 & 0.42 & 0.79 \\
\hline \multicolumn{2}{l}{ Total energy for one measurement } & 2.81 & 4.48
\end{tabular}

\section{B. Mobile application for smartphone}

In order to test the ECG measurement functionality and verify the quality of the collected signals, a smartphone application was developed by using Android OS. This application has a minimum compatibility with Android version 4.3. When the smartphone is held closed to the sensor, the NFC manager triggers an event within Android which launches the application. The application automatically connects to the sensor through the NFC interface and reads the M24LR16E's EEPROM memory. At the same time, energy can be harvested by the device to charge a capacitor, as proposed below. Data transfer usually takes less than 2 seconds, but the phone can be maintained in position close to the device for a few seconds more for additional energy transfer. Upon completion of the data transfer from the memory to the smartphone, the signal is immediately plotted on the smartphone's display. An example of EGC signals acquired with the sensor device is shown on Figure 4. 


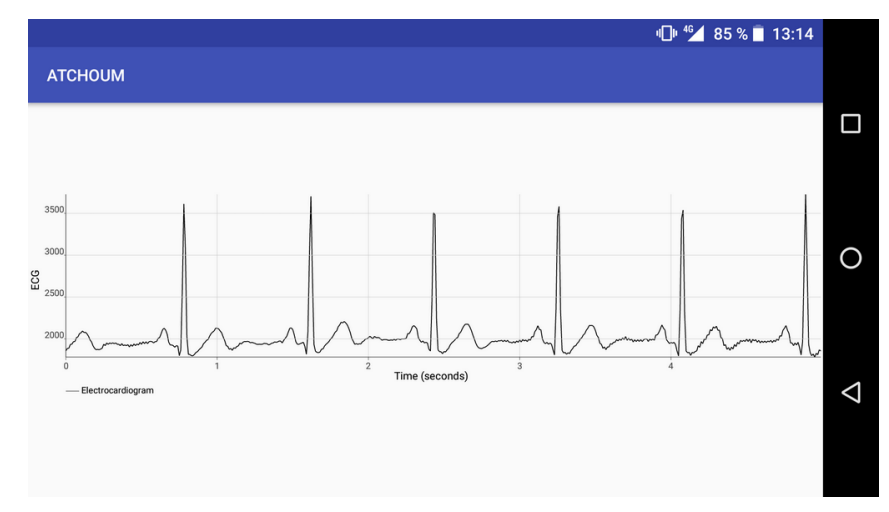

Fig. 4. Example of a 5 second period of ECG acquired by the sensor device and plotted on the smartphone's display.

\section{POWER MANAGEMENT AND ENERGY STORAGE}

An extension board was added to the original sensor board to add the capability of storing energy from the NFC interface in a capacitor, and hence suppling the sensor board in return. To adapt the power management circuit to the output capabilities of the M24LR16E, a current-voltage characterization was performed and is reported on Figure 5. This characteristic was obtained with optimal coupling between both antennas, smartphone's one and the sensor's one (i.e. distance of $5 \mathrm{~mm}$ and parallel antennas).

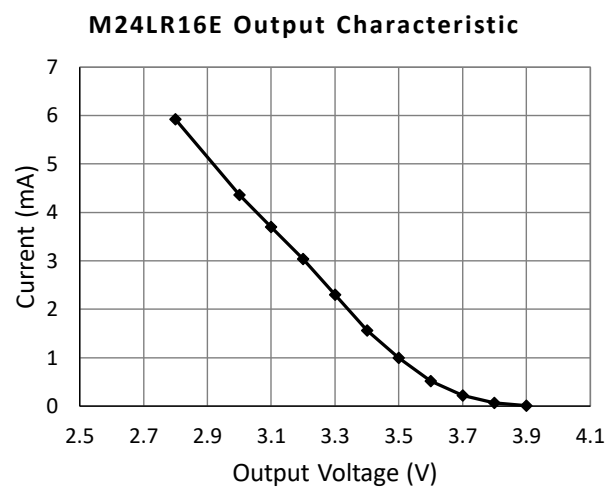

Fig. 5. Measured current-voltage characteristic for the energy harvesting output of the M24LR16E using NFC.

The NFC interface can provide a maximum output of $3.9 \mathrm{~V}$ (in open circuit) and a maximum current of $6 \mathrm{~mA}$. Above this value, the M24LR16E automatically disables this output, so careful consideration must be given when designing the power management unit to avoid this eventuality. The capacitors' charging current can be very high as they act almost like short-circuits when completely discharged, and hence a current limiter must be inserted in the circuit on the output of the M24LR16E. For this purpose, a current source used as a limiter can provide a shorter time constant than a simple series resistance. A comparison was made between a floating current source (LM334) and a resistor on a fully discharged 0.1 Farad super-capacitor. The resistance value used was $560 \mathrm{Ohm}$ to limit the current below $6 \mathrm{~mA}$. The current source was used as a current limiter, with a maximum delivered current given by equation 1, with $T$ the temperature of operation in Kelvin, and $R$ a resistance connected to the LM334 component as shown on Figure 6.

$$
I=\frac{227 \cdot 10^{-6} * T}{R}
$$

To calculate the resistance $R$ to be inserted in the power management circuit, an operating temperature $T$ of $298 \mathrm{~K}$ was considered for a maximum current $I$ of $6 \mathrm{~mA}$. Thus the value of the resistance $R$ is $12 \Omega$ (rounded up).

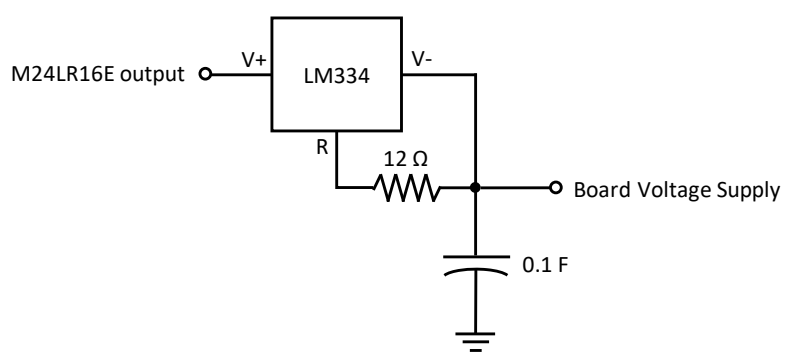

Fig. 6. Diagram of the additional circuit for energy storage from the M24LR16E output with a $6 \mathrm{~mA}$ current limiter.

Both charge characteristics are shown on Figure 7. A minimal voltage of $1 \mathrm{~V}$ across the current source is needed for proper operation, so it cannot charge the capacitor above 3 V. Instead, it has a shorter time constant than the resistor due to its ability to maintain a constant current while the current through the resistance will decrease linearly with the increase in the voltage across the super-capacitor.

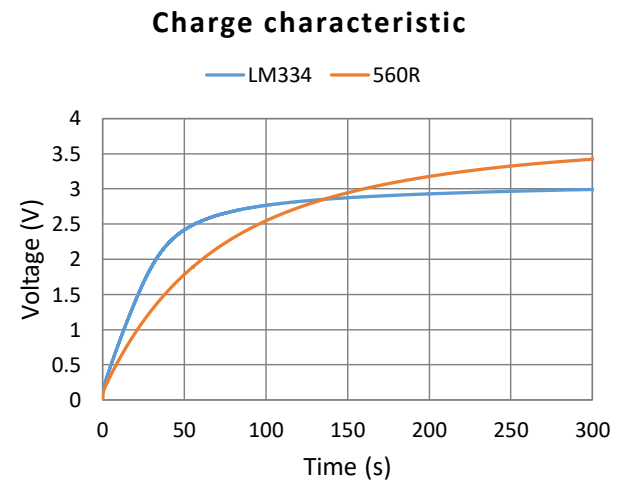

Fig. 7. Measured voltage characteristic when charging the super-capacitor through a fixed resistance of $560 \Omega$ or through a LM334 with a $6 \mathrm{~mA}$ limitation.

As stated, the super-capacitor used for storing the energy has a $5.5 \mathrm{~V}, 0.1 \mathrm{~F}$ capacitance. This value would appear very large at first compared to the energy required for the measurement of a 5-second ECG period. Actually, there are several factors which considerably decrease the efficiency of the power management unit. The first reason is due to the LM334 component. While being used to limit the current with a better efficiency than a resistor (which would give rise 
to a very long time constant during charging), the minimum voltage across the component for proper operation is $1 \mathrm{~V}$. This means that even if the M24LR16E is capable of an output voltage of $3.9 \mathrm{~V}$, the capacitor will not be charged above $2.9 \mathrm{~V}$. In practice, a voltage of up to $3.15 \mathrm{~V}$ has been obtained when charging the super-capacitor with this circuit over 10 minutes. The second limitation is the minimum amount of energy which has to remain in the capacitor in order to maintain a supply voltage above $2.2 \mathrm{~V}$. For example, with a $0.1 \mathrm{~F}$ capacitance, the minimum energy to be stored is $E_{\text {min }}=0.5 \times C \times U^{2}=242 \mathrm{~mJ}$. Thus if the capacitor is charged to $3 \mathrm{~V}$, the energy available for operating the sensor is $E=\left(0.5 \times C \times 3.3^{2}\right)-E_{\min }=303 \mathrm{~mJ}$. This means that only $55.5 \%$ of the energy stored in the capacitor will be available to power the sensor device. A better solution could be to store the energy using a higher voltage, which would enable a reduction of the capacitance for a given amount of energy. Thus the ratio of energy used in the capacitor would be greatly increased. However, many more components would be required for management, such as, for example, a boost converter to raise the output voltage of the M24LR16E, and, conversely, the addition of a buck converter or a low-dropout voltage converter to reduce the voltage of the capacitor for the powering of the sensor board.

\section{CONCLUSION AND FUTURE WORKS}

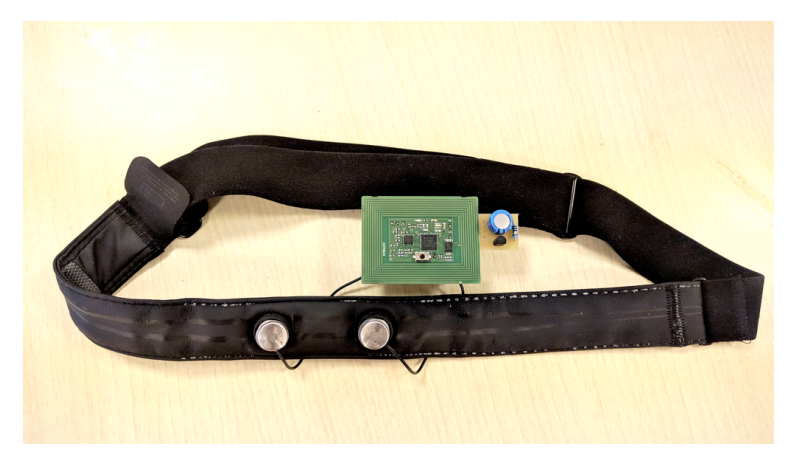

Fig. 8. Illustration of the autonomous sensor system with the power management module, connected to dry electrodes on a chest belt for ECG measurements.

In this paper, a novel sensor system for the ambulatory monitoring of cardiac events has been presented. This device is capable of recording 5-second periods of the ECG and the corresponding samples are stored in an embedded, non-volatile memory. These ECG events can be retrieved at any time using a smartphone with an NFC interface, even if the device is no longer powered. Even when the device is in an idle mode, a low power oscillator is used to clock a real-time calendar function in order to record time and date of the ECG events. Optimizations were made to the hardware design as well as to embedded software to reduce the current consumption down to $800 \mathrm{nA}$ during idle mode with RTC function active, and $160 \mu \mathrm{A}$ when the device is measuring the ECG. The very low power consumption achieved makes possible for the device to be completely self-powered. The device also benefits from a NFC interface enabling the collection of additional energy during the transmission of data to a smartphone. We presented an example of a module for power management and energy storage that can be connected to the sensor device to store the energy collected from the NCF interface into a supercapacitor, thus demonstrating its autonomous capability. The sensor device has been tested in preliminary experiments using standard disposable electrodes for ECG measurements, as well as a chest belt incorporating dry electrodes which can be more conveniently worn over several days (Figure 8). This sensor could be worn on a daily basis without the need to recharge any battery, and be used as a cardiac event monitor without autonomy or memory limitations. Moreover, one can envisage that, in the frame of the mIoT (medical Internet of Things), the ECG events collected with the smartphone could be sent directly to a medical server for real time or postponed analysis by a specialist and thus anticipate cardiovascular events through the detection of early symptoms.

\section{REFERENCES}

[1] V. Feigin et al., "Global, regional, and national life expectancy, all-cause mortality, and cause-specific mortality for 249 causes of death, 19802015: a systematic analysis for the global burden of disease study 2015," The lancet, vol. 388, no. 10053, pp. 1459-1544, 2016.

[2] H. E. Mayhew, "A practical guide to ECG interpretation," Journal of Family Practice, vol. 35, no. 5, pp. 590-592, 1992.

[3] W. B. Kannel, T. Gordon, and D. Offutt, "Left ventricular hypertrophy by electrocardiogram: prevalence, incidence, and mortality in the framingham study," Annals of Internal Medicine, vol. 71, no. 1, pp. 89-105, 1969.

[4] R. Gianrossi, R. Detrano, D. Mulvihill, K. Lehmann, P. Dubach, A. Colombo, D. McArthur, and V. Froelicher, "Exercise-induced st depression in the diagnosis of coronary artery disease. a meta-analysis." Circulation, vol. 80, no. 1, pp. 87-98, 1989.

[5] P. Rautaharju, P. MacInnis, J. Warren, H. Wolf, P. Rykers, and H. Calhoun, "Methodology of ECG interpretation in the dalhousie program; NOVACODE ECG classification procedures for clinical trials and population health surveys." Methods of information in medicine, vol. 29, no. 4, pp. 362-374, 1990.

[6] W. Phanphaisarn, A. Roeksabutr, P. Wardkein, J. Koseeyaporn, and P. Yupapin, "Heart detection and diagnosis based on ECG and EPCG relationships," Medical devices (Auckland, NZ), vol. 4, p. 133, 2011.

[7] D. W. Smith, D. Nowacki, and J. K. Li, "Ecg t-wave monitor for potential early detection and diagnosis of cardiac arrhythmias," Cardiovascular Engineering, vol. 10, no. 4, pp. 201-206, 2010.

[8] J. Anczykowski, S. Willems, B. A. Hoffmann, T. Meinertz, S. Blankenberg, and M. Patten, "Early detection of symptomatic paroxysmal cardiac arrhythmias by trans-telephonic ecg monitoring: Impact on diagnosis and treatment of atrial fibrillation," Journal of cardiovascular electrophysiology, vol. 27, no. 9, pp. 1032-1037, 2016.

[9] B. Massot, T. Risset, G. Michelet, and E. McAdams, "A wireless, lowpower, smart sensor of cardiac activity for clinical remote monitoring," in 2015 17th International Conference on E-health Networking, Application \& Services (HealthCom). IEEE, 2015, pp. 488-494.

[10] PSoC® 4: PSoC 4000S Family Datasheet, Cypress Semiconductor Corporation, July 2017, rev. H.

[11] STM8L151x4, STM8L151x6, STM8L152x4, STM8L152x6 8-bit ultralow-power MCU, STM8L151x4, STM8L151x6, STM8L152x4, STM8L152x6 8-bit ultra-low-power MCU, April 2017, rev. 15.

[12] MSP430FR59xx Mixed-Signal Microcontrollers, Texas Instruments, March 2017.

[13] B. B. Winter and J. G. Webster, "Driven-right-leg circuit design," IEEE Transactions on Biomedical Engineering, no. 1, pp. 62-66, 1983.

[14] Dynamic NFC/RFID tag IC with 16-Kbit EEPROM, energy harvesting, $I^{2} C$ bus and ISO $15693 R F$ interface, ST Microelectronics, August 2017. 